\title{
Movilidad poblacional y procesos de articulación rural-urbano entre los aymara del norte de Chile
}

\section{Population mobility and processes of rural-urban linkages between the Aymara of northern Chile}

\author{
Ana María Carrasco Gutiérrez** \\ Héctor González Cortez ${ }^{* * *}$ \\ Universidad de Tarapacá, Arica, Chile
}

Recibido: 30 de enero de 2014. Aprobado: 23 de noviembre de 2014.

\begin{abstract}
Resumen
Una característica de los aymara del norte de Chile es la movilidad poblacional. Esto no es un suceso reciente, aunque hoy los desplazamientos son más complejos, produciéndose al interior del propio sector rural, como también hacia y desde las ciudades. Con estos movimientos se forman redes complejas y cambiantes por las cuales los individuos circulan dependiendo de sus estrategias de reproducción económica, social y cultural y los acomodos familiares e institucionales que desplieguen. Dentro de este contexto, el objetivo de este estudio es explorar la forma en que el espacio de vida de estos sujetos ya no se limita a lo que acontece en los límites territoriales históricos de la comunidad rural, teniendo una realización translocal, donde las relaciones sociales de sus miembros transitan por un espacio de puntos múltiples y recursos culturales diseminados.
\end{abstract}

Palabras claves: aymaras, movilidad poblacional, translocalidad.

Este trabajo forma parte de los resultados del proyecto Fondecyt № 1110980. "Los significados culturales en los comportamientos y valores sexuales de adolescentes indígenas. Un estudio comparado Aymara-Atacameño". Universidad de Tarapacá, Facultad de Ciencias Sociales y Jurídicas. Departamento de Antropología Además, cuenta con el apoyo del Convenio de Desempeño Universidad de Tarapacá, Ministerio de Educación, MINEDUC.

* Universidad de Tarapacá, Facultad de Ciencias Sociales y Jurídicas, Departamento de Antropología. Correo electrónico amcarrasco@uta.cl

*** Universidad de Tarapacá, Facultad de Ciencias Sociales y Jurídicas, Departamento de Antropología. Correo electrónico hgonzale@uta.cl 


\begin{abstract}
One characteristic of the Aymara people of northern Chile is their population mobility. This is not a recent occurrence, but today the movements are more complex, occurring within the rural sector itself, as well as towards and away from the cities. With these movements, complex and changing displacement networks are formed, by which individuals come and go, depending on their strategies for economic, social and cultural reproduction and family / institutional accommodations. Within this context, the aim of this study is to explore how living space of these individuals is no longer limited only to what happens in the historical boundaries of the rural community because today there exists a translocal embodiment, where the social relations of its members pass through an area of multiple scattered points and cultural resources.
\end{abstract}

Keywords: Aymara, population mobility, translocality.

\title{
Introducción
}

En Chile, la población aymara alcanza un total de 48.501 individuos (Censo 2002), instalados mayoritariamente en las urbes de las regiones de Arica-Parinacota y Tarapacá, lo que ha generado un notorio despoblamiento de sus lugares de asentamiento tradicional (comunidades rurales) producto de la emigración justificada, principalmente, por problemas de pobreza que enfrentan con sus economías de base agropecuaria (González, 2010).

Lo anterior produce preocupación tanto a nivel gubernamental como de la propia sociedad civil, aymaras y estudiosos, ya que se ha interpretado como un proceso de descomposición que generaría funestos efectos tanto a su sociedad como a su cultura (Van Kessel, 1980; 1988). Aspectos concretos que encontramos tras esta imagen son la disminución y envejecimiento de su población rural; las crisis relativamente cíclicas que sufren sus recursos naturales (sequías / inundaciones), que provocan contracciones en la producción agropecuaria; un claro desequilibrio entre la capacidad de sostenimiento productivo del medio y el crecimiento de la población, por los bajos niveles de desarrollo tecnológico existentes; el empobrecimiento de sus economías familiares, vinculado a problemas de articulación mercantil; reproducción social, económica y cultural de comunidades e individuos basada en la realización circunscrita a sus límites territoriales 
históricos; y, finalmente, emigración rural-urbana que implica la salida de sus comunidades de origen (González, 2010; Carrasco y González, 2012a, 2012b).

Este panorama descrito para los aymaras no es nuevo. Numerosos estudios han abordado el tema, indicando que las sociedades campesinas e indígenas, a nivel mundial y desde hace algunas décadas, viven fuertes procesos de urbanización, lo que se constata a través de cifras que indican cómo la mayor parte de esta población radica en ciudades o en sectores distintos a sus antiguos territorios (Kearney, 1996; Levitt y Jaworsky, 2007; Gundermann y González, 2008). También se ha observado que muchos de estos procesos migratorios atraviesan las fronteras de los países, generándose una movilidad de la población que va desde un nivel regional, nacional a mundial, pero con una característica relevante: la persistencia de la relación entre migrantes y comunidades de origen (Loucky y Moors, 2000; Ryang, 2008; Aitchison, Hopkins y Kwan, 2007; Griffith y Savage, 2006). Esta relación trae consigo la creación de múltiples vínculos, generando un fenómeno denominado comunidades transnacionales y comunidades translocales (Vertovec, 2009; Nyberg y Guarnizo, 2007; Guarnizo, 1998; Cohen, 2001), haciendo alusión, fundamentalmente, a la mantención de las identidades de origen de los migrantes, el envío de recursos monetarios a la parentela, la formación de economías étnicas de enclave, diversificación económicamente, etc. (Ratha y Shaw, 2007).

En este artículo proponemos conocer la respuesta que los aymaras han tenido frente a los cambios experimentados en el actual contexto de globalización y modernización; comprender las características de la comunidad aymara actual y demostrar que estamos frente a una vigente comunidad aymara translocal, en la que las redes sociales que la componen abarcan los límites tradicionales históricos pero también los espacios urbanos $^{1}$.

\section{Población aymara y procesos de urbanización}

Al referimos a los aymara y su ubicación territorial, pensamos inmediatamente en sus lugares de asentamiento "tradicional", sin tomar conciencia que esta área de localización espacial ha sido producto de procesos de "arrinconamiento" territorial obligado

1 Como fuentes de información principal se utilizan varios estudios de casos realizados por los autores en comunidades rurales; además, los resultados de dos encuestas (1995 y 2012) aplicadas a una muestra de residentes de origen aymara de las ciudades de Arica e Iquique, cuya finalidad fue recabar antecedentes sobre los procesos migratorios y sus condiciones sociales, económicas y culturales. 
originados por el orden colonial. Esta delimitación ha contribuido a la visión de un área rural aislada y "contenedora de cultura" (González, 2005), donde residen. Inversamente está el espacio urbano, moderno, sin la marginalidad y la pobreza que se asigna a la ruralidad.

El crecimiento económico de las ciudades y pueblos del desierto ha provocado intensas migraciones, en las que se incluyen las áreas indígenas. Poco a poco su presencia urbana se hace visible, aumenta, interpretándose como un tipo de migración interna (rural-urbana).

Ubicados en las ciudades, la vida de los aymara es estudiada a partir de las estrategias de adaptación a esta ${ }^{2}$ y desde los efectos de su marginalidad urbana (pobreza económica, desestructuración social y cultural). Bajo estas aproximaciones, lo rural y lo urbano siguen siendo percibidos como sectores separados, persistiendo la presencia de un lugar tradicional de asentamiento, situado en los antiguos límites territoriales de las comunidades. Por otra parte, los censos corroboran la urbanización de la población indígena ${ }^{3} y$ los estudiosos dan cuenta de dos nuevos fenómenos: primero, que los indígenas urbanos no necesariamente desaparecen culturalmente en las ciudades, sino por el contrario, desde estas protagonizan un revival étnico-cultural; segundo, que ellos siguen ligados a sus comunidades de origen, por lo que los espacios rurales y urbanos se vinculan e integran por relaciones sociales, culturales y económicas. Como resultado aparece el concepto de movilidad de la población indígena, que ayuda a comprender mejor la migración campo-ciudad, cuestionando el sentido unidireccional tradicionalmente atribuido (Redfield, 1947) y abriendo la mirada a una práctica donde existe un constante flujo y reflujo de personas dentro del mismo sector rural y entre este y las áreas urbanas.

Para el caso aymara, la influencia de los emigrados en las comunidades de origen, la importancia de estas en los migrantes, así como los movimientos de personas, bienes, recursos y capital social y cultural entre estos espacios, reafirman la tesis que para comprender y analizar hoy sus formas de vida es necesario relacionar campo y ciudad, explorar no solo los vínculos actuales entre esos dos mundos, sino también profundizar en su historia (Alber, 1999 [1990]; Paerregaard, 1997, 1998, 2000; González, 2007, 2010).

2 Organización de los migrantes para conseguir servicios urbanos en las barriadas marginales, la creación de centros de residentes o de mecanismos de adaptación transicional.

3 De acuerdo a datos elaborado a partir de CELADE, Fondo Indígena, 2008, tenemos que en Chile la población indígena comprende un total de 692.192 (4,6\% de la población nacional), de los cuales 243.810 individuos viven en el área rural y 448.382 en la urbana, alcanzando la población indígena urbana un 64,8\% (a nivel latinoamericano el porcentaje es de 40,0\%) (http://celade.cepal.org/redatam/PRYESP/SISPPI). 


\section{Población aymara, distribución espacial y patrón de agrupamiento residencial}

En el Censo 2002 se contabilizaron en Chile 48.501 personas que se declararon aymaras. De estas, la mayor parte, un $84,4 \%$, reside en la XV y I regiones (40.934), distribuidas en el sector urbano con 31.033 habitantes, principalmente en las ciudades de Arica: 23.192 e Iquique: 9.064; y en el rural, con 9.901 individuos.

Centrándonos solo en la población rural, tenemos que esta se reparte, según sectores geográficos, de la siguiente manera. En el altiplano se ubica aproximadamente un 23,7\% (comunas de General Lagos, parte de la comuna de Putre, Colchane y parte de la comuna de Pica). En el sector precordillerano o de valles altos se ubica un 33,6\% (comunas de Putre, Camarones, Camiña, Huara y Pozo Almonte). En el sector de valles bajos (comunas de Arica, Camarones, Camiña, Huara, Pozo Almonte y Pica) se ubica el 42,7\%.

Respecto al patrón de agrupamiento residencial, este depende de la zona que se trate y está relacionado con la orientación económica del área donde se ubiquen las comunidades. Así, en el altiplano es disperso, ya que las familias residen en las estancias, unidad territorial donde tiene viviendas el grupo familiar y donde pastorean sus rebaños. La comunidad está compuesta por varias estancias. Aunque todos tienen casa en el poblado central de la comunidad, los comuneros acuden a este solo cuando tienen reuniones sociales o festivas. En los valles el patrón residencial es nucleado, ya que todos viven en un poblado central que está rodeado por los sectores de cultivo, lugar donde los comuneros se dirigen muy temprano en la mañana para realizar sus tareas agrícolas, retornando al atardecer.

La mayor cantidad de población se concentra en la zona parcelaria de constitución reciente y económicamente más dinámica. En la zona "comunitaria" las densidades de población son muy bajas, siendo las localidades con mayor cantidad de población las económicamente más dinámicas. Sin embargo, existen algunas excepciones, como por ejemplo en el altiplano, donde esta característica puede estar relacionada con la presencia original de más de un patrilinaje, un patrilinaje demográficamente fuerte o la constitución de una estancia como centro político o de servicios. También se observa en los valles, con la presencia de una mayor superficie de cultivo y, por ende, de un mayor número original de familias. 


\section{Comunidades aymara rurales y cambios demográficos}

Datos censales muestran que, a partir de la década de 1950, la población de las comunidades rurales aymaras, residentes en las actuales XV y I región, empieza a disminuir drásticamente producto de la emigración hacia las ciudades. Junto con esto se observan cambios en la estructura de edad, apreciándose un claro "envejecimiento" debido a que la emigración afecta fundamentalmente los estratos que van entre los 15 y los 50 años, edades atractivas para las estructuras de empleo urbanas. Lo interesante de esta situación es que hasta hoy se siguen manteniendo similares características en cuanto al número de habitantes y las particularidades de la estructura demográfica, sin que se produzca un colapso demográfico debido, fundamentalmente, a que desde las ciudades está regresando población a estas comunidades, como asimismo porque está siendo reemplazada por personas que descienden desde áreas más altas (Carrasco y González, 2012a; 2012b). Específicamente, la población rural que se mantiene en sus comunidades de origen está constituida por individuos en edad no atractiva para los mercados laborales urbanos, tales como jubilados que regresan de la ciudad y/o matrimonios jóvenes que deciden permanecer. También están los individuos que llegan desde los sectores más altos (incluidos países vecinos) para insertarse en el mercado de tierras en aparcería e incluso venta, que entregan los migrantes que siguen manteniendo predios en su poder.

Tenemos entonces un flujo y reflujo constante de personas dentro del mismo sector rural y las áreas urbanas, por motivaciones sociales, culturales y económicas. Las características de esta movilidad demuestran la capacidad de adaptación de los aymaras chilenos a los nuevos contextos que enfrentan. Con todo, hay que destacar que esto no ocurre solo a partir de la atracción urbana, ya que antiguamente también se desplazaban para intercambiar o vender su producción, comprar lo requerido para su subsistencia, trabajar por productos o dinero en otros sitios, realizar trámites, etc. La diferencia es que por entonces se mantenían con sus familias en las comunidades de origen.

Así, vemos que los aymaras del norte de Chile han hecho de la anormalidad demográfica, al no mantener la clásica pirámide de edades sino una con menos población entre los 15 y los 50 años, producida por los impactos económicos y sociales de la emigración a las ciudades, un ajuste estructural que permite la supervivencia cultural de la misma comunidad rural. Ahora, esta pérdida de población rural debe entenderse como parte de una estrategia estructural que se explica conociendo la prolongación de las comunidades en la ciudad; incorporando en el análisis el hecho que el espacio de vida comunal ya no se limita o circunscribe a lo que acontece en los límites territoriales de la comunidad de asiento rural (González, 2010). 


\section{Economía y comunidad}

Pese a que existen motivos objetivos ${ }^{4}$ y subjetivos ${ }^{5}$ que justifican la migración de los aymaras rurales, también son fundamentales ciertas características de los sistemas de producción que permiten la movilidad de las personas. Una de ellas es la disminución de la demanda de fuerza de trabajo durante ciertos períodos del ciclo productivo agropecuario anual.

En el altiplano, la ganadería extensiva no requiere de gran inversión de fuerza de trabajo, ya que las labores se limitan principalmente a vigilar los animales, tareas que realizaban normalmente las mujeres y los niños de ambos sexos y que, actualmente, han quedado a cargo de personas mayores. En la precordillera, en tanto, existen restricciones climáticas que limitan la producción agrícola durante el período invernal, ya que el ciclo de cultivos anuales se realiza entre septiembre y mayo, por lo que durante junio y agosto existe menor requerimiento de trabajo; respecto a la ganadería, su importancia ha ido disminuyendo notoriamente en las últimas décadas, siendo cambiada por cultivos comerciales (especialmente ajo y orégano). En los sectores más bajos, donde se realizan cultivos durante todo el año en los valles frutícolas, aparte del regadío y ciertos cuidados puntuales, la mayor demanda de trabajo se produce durante la etapa de cosecha, que se concentra entre enero y mayo. En todos los sectores agrícolas de valles, el tamaño más bien pequeño de los predios hace que no se demande gran cantidad de mano de obra durante los momentos más exigentes del ciclo productivo anual.

Los períodos de "ocio" en el ciclo productivo agropecuario permiten liberar a parte de los miembros del hogar para el desempeño de otras ocupaciones o actividades en otros lugares ${ }^{6}$. En la misma dirección apuntan una serie de acomodos destinados a satisfacer los requerimientos de fuerza de trabajo permanentes, como el pastoreo en el

4 Las crisis relativamente cíclicas de los recursos naturales (como sequías e inundaciones), que provocan contracciones en la producción agropecuaria. Un evidente desequilibrio entre la capacidad de sostenimiento productivo del medio y el crecimiento de la población, dados los niveles de desarrollo tecnológico existentes. La pauperización de sus economías familiares por condiciones desfavorables de articulación mercantil.

5 La causal subjetiva más importante para la emigración es el acceso a educación, propia o para los hijos. Las nuevas generaciones migran a otros lugares, especialmente las ciudades, para seguir estudiando y obtener mayor nivel educacional que el que pueden encontrar en el sector rural ( $6^{\circ}$ básico). La búsqueda de mayores niveles educativos operó en un primer momento para los hijos varones, pero en la actualidad es común a ambos sexos. Las generaciones más jóvenes de aymaras urbanos han igualado los niveles promedios de escolaridad que presentan los no aymaras de la misma edad.

6 La excepción la constituyen ciertas áreas de valles más dinámicos económicamente, como Camiña, donde se combina en un mismo predio la producción de ajo y maíz-choclo, por lo que la demanda de trabajo es mayor y más permanente. Lo mismo puede ocurrir cuando se activan coyunturalmente ciertos sectores, como ocurrió 
altiplano o el regadío y otras tareas menores en los valles, que se dejan a cargo de personas de mayor edad. Estos arreglos tienen que ver, normalmente, con la separación de los grupos familiares que mantienen sistemas de doble e incluso triple residencia, según los diferentes espacios donde mantengan intereses (González, 1990). En otras ocasiones se recurre a parientes, vecinos o directamente a la contratación esporádica de peones que viven en la propia localidad o que llegan de otras partes, generalmente de Bolivia.

Otro factor que facilita la movilidad lo constituye la mantención de bienes en las comunidades de origen, aun después de instalarse en otros sitios. En el altiplano, si bien el manejo de los rebaños es familiar, los animales son de propiedad individual ${ }^{7}$. Producto de esta situación, en las comunidades existen rebaños que normalmente incluyen animales de individuos que han emigrado. En este tipo de arreglos son importantes padres y abuelos que, generalmente, se mantienen en el lugar. En el caso de los valles, los catastros de propiedad señalan que buena parte de los propietarios reside fuera de la localidad, principalmente en las ciudades, acudiendo a la comunidad solo en épocas de trabajo agrícola, para lo cual también pueden llegar a acuerdo con parientes o vecinos (González, Gundermann y Rojas, 1991). Algunos entregan los terrenos a terceros mediante arriendos o acuerdos de mediería; como asimismo, otros recurren a convenios tales como cesiones o custodia temporales que operan, mayormente, entre parientes cercanos (Carrasco y González, 2012a).

La movilidad también se relaciona con las variaciones en los precios de los productos o el aprovechamiento de oportunidades de mercado. Un ejemplo claro lo constituyó la introducción del orégano en sectores de precordillera (Belén) y el ajo en el altiplano (Chulluncane). También encontramos una situación similar en los años 80, con la importancia que en este período adquirió la venta de carne de camélido en las ciudades, durante la crisis económica que vivió el país. Ahora, estas posibilidades de ventas que abre el mercado pueden modificarse negativamente para los productores rurales y aca-

con el cultivo del ajo en comunidades del sector altiplánico en la comuna de General Lagos, que fue introducido por la posibilidad de cosechar en temporada distinta a la de los valles.

7 Los rebaños se constituyen a partir de regalos de hembras que realizan los padres y eventualmente los padrinos a los niños, hombres y mujeres, con ocasión de la ceremonia del "floreo" de animales (waiño o qillpa), "cortes de pelo" (ceremonia asociada al bautismo o imposición de nombre) u otros momentos no rituales. Los animales de los niños se mantienen en el rebaño de sus padres, aumentando su número por reproducción, regalos matrimoniales u otras donaciones. Por efectos de la residencia patrilocal, normalmente el ganado de los miembros del linaje o sublinaje se mantienen en rebaño colectivos. Solo se segmenta cuando una mujer se separa del grupo familiar para residir junto a su esposo y, al cabo de un tiempo, lleva sus animales y los incorpora a la tropa de su marido. Este fenómeno también ocurre cuando las unidades de producción familiar se independizan completamente de los padres, lo que ocurre comúnmente cuando ellos ya son muy ancianos o han fallecido. 
rrear procesos inversos, como el abandono de las comunidades, una contracción en los volúmenes de producción y de los ingresos agroganaderos ${ }^{8}$.

Los ejemplos mencionados enseñan no solo la complejidad de los movimientos poblacionales sino también las estrategias económicas, con la activación o contracción de la producción agropecuaria, que realizan los aymaras.

Actualmente, estudios realizados (González, 2010; Carrasco y González, 2012a, 2012b) nos muestran cómo la producción agropecuaria, proveniente de la explotación predial, no explica la reproducción económica de las familias comuneras, ya que solo representan alrededor de la mitad de sus ingresos totales. Sin embargo, esta tendencia se revierte cuando existe una mejor articulación con el mercado. Parafraseando a González (2010), únicamente cuando no existen oportunidades rentables de mercado para sus productos la familia contrae sus niveles de producción agropecuaria a niveles de autoconsumo y recurre, además, a sistemas de reproducción económica de mayor complejidad y diversidad. Con lo anterior queremos decir que cuando más vinculados parecen estar con las ciudades y los procesos de modernización, más se orienta la producción agropecuaria al autoconsumo. El incremento del autoconsumo debe relacionarse más bien con las dificultades que encuentran para la colocación de sus producciones en los mercados locales y regionales, en las restricciones ambientales y culturales para aumentar sus volúmenes de producción o introducir nuevos cultivos y tecnología, así como la falta de capital y de políticas gubernamentales más efectivas de asistencia y apoyo productivo. Sin embargo, no estamos frente al regreso a una economía de autosubsistencia, puesto que los hogares procuran obtener entradas monetarias por otras vías, lo que se advierte en la importancia de los ingresos extraprediales, que aumentan en monto como en diversidad (González, 1997; 1996a; 1996b).

La gran importancia que han adquirido los ingresos extraprediales dentro de la composición total de las entradas familiares se debe a que frente a la imposibilidad de aumentar su producción agropecuaria, por condiciones estructurales internas o por ausencia de mercado atractivo, esta se contrae o se mantiene a límites de consumo, pero no necesariamente se abandona la comunidad, sino que se incorporan otras actividades e ingresos que se consiguen fuera del predio y se realizan los acomodos familiares o sociales pertinentes para mantener la explotación predial.

8 Por ejemplo disminución en los precios por la aparición de competencia, como aconteció con el orégano y la carne camélida; o problemas de plagas, como ocurrió con el ajo. 
Las actividades e ingresos no agropecuarios se han hecho mucho más diversos. La práctica del trabajo temporal no es nueva, constituyéndose en una experiencia habitual, un par de décadas atrás, que personas del altiplano bajaran a zonas de valles a emplearse en los períodos de mayor demanda de trabajo del ciclo agrícola, aunque muchas veces lo hacían por productos; lo mismo hacían acudiendo a trabajar en comunidades bolivianas vecinas, de donde traían quínoa o papas. Los habitantes de los valles, en tanto, podían emplearse en otras zonas más dinámicas, en oficinas salitreras de la pampa o en las ciudades; la diferencia actualmente es que, aparte de ser más frecuente, ha aumentado su radio de alcance dentro del mismo sector rural y en las ciudades, se realiza ahora casi enteramente bajo patrones monetarios y ya no principal o exclusivamente en la agricultura. El abanico de posibilidades es mayor, incluso, en las mismas zonas rurales, donde se ha sumado el empleo periódico en obras viales, de infraestructura hidráulica o construcciones que realiza el gobierno. En las mismas comunidades también pueden encontrar algunos puestos estables de trabajo ofrecidos por los servicios públicos. Actualmente, también es usual que algunos miembros del hogar se empleen en otros sitios como la ciudad ${ }^{9}$, sin olvidar que la mayor parte de los habitantes del sector rural ahora tiene casa en los sectores urbanos de la región, gracias a los programas estatales de construcción de viviendas sociales. También han adquirido mayor importancia las ocupaciones por cuenta propia, tales como el comercio y el transporte (Carrasco, 1998).

En relación con la mayor vinculación de las familias con el mundo urbano, poco a poco han ido adquiriendo gran importancia los ingresos correspondientes a aportes de terceros que, en general, corresponden a remesas en dinero o mercaderías que periódicamente envían hijos u otros familiares que viven en las ciudades. Entre la población originaria de los valles, especialmente de los más bajos (correspondientes a las comunas de Arica, Camarones, Camiña, Huara, Pozo Almonte y Pica), también aumenta la importancia de los pagos por jubilaciones. Uno de los rubros de ingreso monetario más significativo lo constituyen los subsidios estatales que se entregan por asignaciones a menores y pensiones a ancianos y discapacitados. Según estudios realizados en la zona (González, 2010), son estos subsidios en dinero los que han tenido un mayor impacto como factor de retención de población en los espacios rurales. Ellos constituyen un mecanismo de consecución monetaria que se podría relacionar justamente con las estructuras demográficas "anormales" que presentan las comunidades, en la medida que quienes no los reciben son, precisamente, las personas ubicadas en los rangos de edad más impactados por la emigración.

9 Ocurre así, por ejemplo, con hijos que con mayores niveles de educación y experiencia urbana trabajan en la ciudad y retornan a la comunidad en períodos de cesantía. 
La obtención de dinero se relaciona con el comportamiento del gasto de las unidades familiares aymaras. Así, dentro del consumo alimenticio actualmente tienen gran importancia los artículos de procedencia externa, lo que reafirma el fuerte proceso de monetarización del consumo que ya se hacía sentir años antes. Otros cambios dicen relación con la relevancia que han asumido los costos derivados de la prolongación urbana de los hogares; como asimismo el que los hogares han debido incorporar nuevas inversiones en sus comunidades, producto del mejoramiento del acceso a servicios básicos a la vivienda, como luz y agua. Por otra parte, comparado con lo que acontecía antiguamente, hoy se observa un aumento de la incidencia de los gastos en educación y en transporte. Los primeros se relacionan con una fuerte demanda por mayores niveles educacionales, que afecta a grupos familiares encabezados por parejas más jóvenes, con hijos estudiando en la ciudad, que deben destinar recursos obtenidos en el sector rural para financiar su inserción urbana. Los segundos, con el aumento de los viajes hacia otras localidades y centros urbanos, dada la alta movilidad que hoy presentan las familias.

\section{Conclusiones}

Los antecedentes presentados demuestran que la población aymara que reside en el sector rural vive actualmente un ajuste estructural producto de una nueva realidad, caracterizada por la poscomunalidad ${ }^{10}$, lo que se evidencia a través de la disminución de la población con residencia permanente y la modificación de las estructuras de edades que generan una anormalidad en ellas.

Ahora, lo interesante es que no se trata de una crisis poblacional y menos de un colapso demográfico que genere la representación de una comunidad en crisis o en proceso de descomposición; tampoco estamos frente a comunidades incompletas, terminales o agonizantes, simplemente ellas se completan con sus derivaciones hacia otras zonas del sector rural y, principalmente, hacia las ciudades y áreas suburbanas. La comunidad ya no se reproduce dentro de sus límites territoriales históricos, ya que las redes económicas, sociales y culturales que le dan sentido traspasan sus antiguas fronteras. El espacio comunal se prolonga hacia los distintos sitios donde se encuentren dispersos o transiten sus miembros. Actualmente las redes sociales de la comunidad aymara tienen una rea-

10 Noción que hace referencia a cambios concernientes a la desruralización generada por las emigraciones y movilidad; cambio en el patrón de ingresos, ya no netamente agrario; descampesinización de la organización económica; y translocalización de los patrones de relaciones sociales. Para mayor información ver Gundermann y González, 2008. 
lización translocalizada, con individuos y recursos culturales diseminados por puntos múltiples y, además, móviles.

Ya no existen las comunidades aisladas, cerradas e internamente homogéneas que caracterizaron por mucho tiempo al mundo aymara. Esto tanto porque ahora vemos comunidades con un mayor nivel de heterogeneidad, dedicadas incluso a actividades agropecuarias comerciales, sino fundamentalmente porque la imagen del mundo andino confinado a las zonas rurales, reproduciéndose dentro de los límites de la comunidad local, con escaso contacto con el mercado, desvinculada de la sociedad y la cultura nacional, no opera. La desterritorialización, los procesos migratorios, los conflictos de poder y otros cambios sociales que han experimentado las comunidades rurales impiden seguir esencializando lo andino en virtud de su supuesto exotismo.

Asimismo, los antecedentes que se manejan nos muestran cómo la economía familiar de los residentes en las zonas del interior no se puede caracterizar solo a partir de su condición de pequeños productores agropecuarios, ya que hoy estamos frente a nuevo tipo de 'campesino' que se caracteriza por su alto nivel de diversificación económica, que puede ser beneficiario de los subsidios monetarios estatales, trabajador asalariado, artesano, comerciante, transportista, etcétera. No se trata de sujetos condenados a vivir de la producción agropecuaria. Ahora, esta estrategia no se puede comprender en y desde la comunidad rural local, ya que las actividades económicas también se han translocalizado e incluyen los espacios urbanos. De esta manera, las comunidades aymaras rurales no pueden seguir siendo consideradas como entidades opuestas a las ciudades, cuya relación se centra en la adquisición de bienes y servicios no autoproducidos, la venta de sus producciones agrícolas o la emigración cuando falla la capacidad de sostenimiento productivo local.

En definitiva, la desterritorialización de las antiguas comunidades indígenas o campesinas rurales ha dado lugar a complicados procesos de articulación de los sectores rurales y urbanos. En el caso de los aymaras del norte de Chile, la movilidad poblacional ha adquirido tal complejidad que ha puesto en cuestión la posibilidad de seguir tratando los sectores rurales y urbanos como construcciones teóricas demográfica y socialmente discontinuas.

Actualmente el espacio de vida de estos sujetos indígenas ya no se circunscribe a lo que acontece en los límites territoriales históricos de la comunidad rural o en los sitios urbanos hacia los que hayan migrado, sino a la interrelación social, económica y cultural entre lo rural y lo urbano, en el despliegue de una nueva comunidad translocalizada, en 
la cual sus estructuras demográficas, la movilidad y el comportamiento económico de sus miembros, refieren solo a un ajuste estructural vinculado a la emergencia de este nuevo tipo de comunidad.

\section{Referencias}

Aitchison, C., Hopkins, P. E. y Kwan, M. (Eds.) (2007). Geographies of Muslim Identities: Diaspora, Gender and Belonging. Ohio, Estados Unidos: Ashgate Publishing, Ltd., Ohio State University.

Alber, E. (1990). ¿Migración o movilidad en Huayopampa? Nuevos temas y tendencias en la discusión sobre la comunidad campesina en los Andes. Lima, Perú: Instituto de Estudios Peruanos.

Carrasco, A. y González, H. (2012a). La movilidad poblacional aymara en tiempos de poscomunalidad. Ponencia presentada en el XXXIV Convegno Internazionale di Americanistica, 3-10 de mayo. Perugia, Italia.

-___. (2012b). Movilidad poblacional: un ajuste estructural a la nueva realidad poscomunal de los aymara del norte de Chile. Ponencia presentada en el Tercer Congreso Latinoamericano de Antropología (ALA), 5-10 de noviembre. Santiago, Chile.

Carrasco, A. M. (1998). Mujeres aymara e inserción laboral. Revista de Ciencias Sociales, 8, 83-96.

Cohen, J. (2001). Transnational migration in rural Oaxaca, Mexico: Dependency, development, and the household. American Anthropologist, 103 (4), 954-967.

González, H. (1990). Timar, diagnóstico socioeconómico. Acerca de la utilización de dos espacios económicos: el campo y la ciudad. Serie Documentos de Trabajo. Arica, Chile: Taller de Estudios Andinos.

-_- (1996a). Características de la migración campo-ciudad entre los aymaras del norte de Chile. Serie Documentos de Trabajo. Arica, Chile: Corporación Norte Grande.

. (1996b). Los migrantes aymaras en la ciudad: acceso a educación, vivienda y salud. Serie Documentos de Trabajo. Arica, Chile: Corporación Norte Grande. 
- (1997). La inserción económica de los migrantes aymara en la ciudad: el trabajo como empresa familiar y la reproducción cultural. En Actas Segundo Congreso Chileno de Antropología, Tomo I (pp. 315-324). Santiago, Chile: Colegio de Antropólogos de Chile.

-__- (2005). ¿Están las comunidades aymaras del norte de Chile en crisis? Una respuesta desde la demografía y la economía. Manuscrito en posesión del autor.

- (2010). Comunidad rural en crisis o comunidad translocalizada entre los aymara del norte de Chile. En Miradas desde el Sur. Actas del VI Congreso Chileno de Antropología, Tomo I (pp. 538-551). Santiago, Chile: LOM ediciones.

González, H. y Gundermann, H. (1989). Campesinos y aymaras en el norte de Chile. Serie Documentos de Trabajo. Arica, Chile: Taller de Estudios Andinos.

González, H., Gundermann, H. y Rojas, R. (1991). Diagnóstico y estrategia de desarrollo campesino en la I región de Tarapacá. Serie Documentos de Trabajo. Arica, Chile: Corporación Norte Grande.

Gundermann, H. y González, H. (2008). Pautas de integración regional, migración, movilidad y redes sociales en los pueblos indígenas de Chile. Universum, 23 (1), 82-115.

Guarnizo, L. E. (1998). The rise of transnational social formations: Mexican and Dominican state responses to transnational Migration. Political Power and Social Theory, 12, 45-94.

Griffith, R. M y Savage B. D. (Eds.) (2006). Women and religion in the African diaspora: knowledge, power, and performance. Baltimore, Estados Unidos: Johns Hopkins University Press.

Instituto Nacional de Estadísticas INE, Programa Orígenes MIDEPLAN-BID (2005). Estadísticas sociales de los pueblos indígenas en Chile, Censo 2002. Santiago, Chile: Instituto Nacional de Estadística.

Kearney, M. (1996). Reconcentualizing the peasantry: Anthropology in global perspective. Colorado, Estados Unidos: Westview Press.

Levitt, P. y Jaworsky, B. N. (2007). Transnational migration studies: Past developments and future trends. Annual Review of Sociology, 33, 129-156. 
Loucky, J. y Moors, M. (Eds.) (2000). The Maya Diaspora: Guatemalan Roots, New American Lives. Philadelphia, Estados Unidos: Temple University Press.

Nyberg Sorensen, N. y Guarnizo, L. E. (2007). La vida de la familia transnacional a través del Atlántico: la experiencia de la población colombiana y dominicana migrante en Europa. Puntos de vista. Cuaderno del Observatorio de las Migraciones y de la Convivencia Intercultural de la Ciudad de Madrid, 9, 7-30.

Paerregaard, K. (1997). Linking separate worlds: urban migrants and rural lives in Peru. Oxford, Gran Bretaña: Berg Publishers.

____ (1998). The dark side of the moon. Conceptual and methodological problems in studying rural and urban worlds in Peru. American Anthropologist, 100 (2), 397-408.

____ (2000). Procesos migratorios y estrategias complementarias en la sierra peruana. European Review of Latin American and Caribbean Studies, 69, 69-80.

Ryang, S. (2008). Writing Selves in Diaspora: Ethnography of Autobiographics of Korean Women in Japan and the United States. Lexington, Estados Unidos: Lexington Books.

Ratha, D. y Shaw, W. (2007). South-South Migration and remittances. The World Bank working paper, 102. Washington, D. C., Estados Unidos: Banco Mundial.

Redfield, R. (1947). The Folk Society. American Journal of Sociology, 52 (4), 293-308.

Van Kessel, J. (1980). Holocausto al progreso. Los aymaras de Tarapacá. Amsterdam, Holanda: Centro de Estudios y Documentación Latinoamericana.

- (1988). Los aymaras contemporáneos Chile (1879-1985). Su historia social. Cuadernos de investigación social. Iquique, Chile: CREAR.

Vertovec, S. (Ed.) (2009). Anthropology of Migration and Multiculturalism: New Directions. Londres, Inglaterra: Routledge. 